\title{
Liderança coaching na enfermagem e sua influência na satisfação profissional e segurança do paciente
}

\author{
Nursing coaching leadership and its influence on job satisfaction and patient safety \\ Liderazgo coaching en la enfermería y su influencia sobre la \\ satisfacción profesional y seguridad del paciente
}

Como citar este artigo:

Moraes MCS, Dutra GO, Ferreira TDM, Dias FCP, Balsanelli AP, Gasparino RC. Nursing coaching leadership and its influence on job satisfaction and patient safety. Rev Esc Enferm USP. 2021;55:e03779. https://doi.org/10.1590/S1980-220X2020042103779

\section{Márcia Cristina Souza de Moraes ${ }^{1}$ \\ Gabrielle Oliveira Dutra ${ }^{2}$ \\ Thelen Daiana Mendonça Ferreira ${ }^{2}$ \\ Flávia Carvalho Pena Dias ${ }^{2}$ \\ Alexandre Pazetto Balsanelli ${ }^{3}$ \\ Renata Cristina Gasparino ${ }^{2}$ \\ ${ }^{1}$ Universidade Estadual de Campinas, Hospital de Clínicas, Campinas, SP, Brasil. \\ ${ }^{2}$ Universidade Estadual de Campinas, Faculdade de Enfermagem, Campinas, SP, Brasil. \\ ${ }^{3}$ Universidade Federal de São Paulo, Escola Paulista de Enfermagem, São Paulo, SP, Brasil.}

\begin{abstract}
Objective: To compare the nursing technicians' perception with the nurses' self-perception of the exercise of nurses' coaching leadership and to check the influence of this leadership model on the safety climate and on the team's satisfaction. Method: This is a correlational study, carried out with 85 nurses and 85 nursing technicians, using the Questionnaire on the Nurse's Self-Perception of Leadership Exercise, the Questionnaire on Nursing Technicians' and Assistants' Perception of Leadership Exercise, and the subscales Safety climate and Satisfaction at Work. The relations among the professionals' responses were assessed using the Mann Whitney test and Spearman's coefficient. Results: Nurses achieved higher means in the four dimensions of coaching Leadership, and in three, the differences were significant $(p<0.05)$. The dimensions of Coaching Leadership obtained positive and significant correlations with the subscales Safety Climate and Satisfaction, the majority being of moderate magnitude. Conclusion: Nurses'self-perception regarding the exercise of leadership was more positive than the technicians' assessment. The more the nurse exercises the dimensions of Coaching Leadership, the better the team's safety climate and satisfaction.
\end{abstract}

\section{DESCRIPTORS}

Leadership; Nursing, Supervisory; Job Satisfaction; Patient Safety; Nurse's Role; Health Services Administration. 


\section{INTRODUÇÃO}

O conceito de liderança é multidimensional e complexo. Dentre as várias tentativas de elucidar o termo, autores apontam que a liderança é a "capacidade de influenciar um conjunto de pessoas para alcançar metas e objetivos" ${ }^{\text {"(1). }}$.

$O$ exercício da liderança em enfermagem tem influência na organização do trabalho em saúde e concretiza-se na interação da equipe de enfermagem e da equipe multidisciplinar, tendo como foco a assistência ao indivíduo e sua família.

Nesse sentido, as Diretrizes Curriculares Nacionais determinam que a liderança, considerada uma competência central, deve ser desenvolvida desde a graduação ${ }^{(2)}$, pois, além de proporcionar maior resolubilidade ao processo de trabalho gerencial, possibilita ao enfermeiro desempenhar com mais propriedade a figura de articulador e motivador da equipe $\mathrm{e}^{(3-4)}$.

$\mathrm{O}$ enfermeiro deve assumir uma postura que transmita confiança, devendo pautar as relações de trabalho considerando que liderar não é somente delegar tarefas, mas também buscar um ambiente de trabalho que favoreça o desenvolvimento profissional e pessoal de seus liderados ${ }^{(5)}$.

Nesse contexto, pesquisadores têm demonstrado relação entre modelos de liderança contemporâneos e melhores resultados referentes à segurança do paciente e à satisfação dos profissionais, fatores esses que podem contribuir para a melhoria da qualidade da assistência ${ }^{(6-7)}$. Dentre esses modelos de liderança, têm sido apontadas na literatura a Liderança transacional ${ }^{(8)}$, Liderança transformacional ${ }^{(9)}$, Liderança autêntica ${ }^{(10)}$, e a Liderança coaching, sendo essa última pautada por um processo de promoção e estímulo da aprendizagem, com orientação prática em busca do alcance de metas relacionadas ao desenvolvimento técnico e pessoal ${ }^{(11-12)}$.

O modelo de Liderança coaching destaca-se no setor empresarial e vem ganhando espaço na área de saúde, tanto na esfera particular quanto pública, pois tem como meta melhorar o desempenho das equipes de trabalho(13-14). Esse modelo de liderança é visto como uma importante estratégia e como um propulsor do desenvolvimento de competências, além de estímulo ao autoconhecimento dos liderados, tornando-se então um acelerador de resultados. Portanto, "a Liderança coaching é considerada um novo direcionamento das organizações do setor de saúde e inovadora entre os enfermeiros brasileiros, sendo oportuno e necessário estudá-la em nossa realidade" ${ }^{\prime(15)}$.

As dimensões do processo coaching incluem Comunicação, Dar e receber feedback, Dar poder e exercer influência e Ofertar apoio à equipe para a obtenção dos resultados conjuntos, sendo aspectos que se refletem nas competências necessárias para o exercício do enfermeiro-líder ${ }^{(16)}$.

No contexto da enfermagem, estudos de Liderança coaching trazem consigo uma metodologia inovadora que propõe uma avaliação correlacional da percepção da liderança entre líderes e liderados ${ }^{(3,13,15)}$. Resultados mostram que o exercício desse modelo de liderança traz melhorias significativas para o aumento da produtividade, e, inclusive, da disposição da equipe para cooperar ${ }^{(5)}$, satisfação no trabalho ${ }^{(3)}$, interação contínua entre enfermeiro e técnico de enfermagem e busca pelo desenvolvimento profissional e pessoal ${ }^{(13)}$.
Em um panorama geral, a liderança coaching na enfermagem apresenta estudos tanto na atenção primária quanto na atenção hospitalar, sendo a segunda com um número significantemente maior de publicações. Na maioria dos estudos é abordada a partir do princípio de ser uma ferramenta utilizada no desenvolvimento da competência de liderar para enfermeiros líderes ${ }^{(3,15)}$, além de avaliar o impacto desse modelo de liderança na equipe de enfermagem, relacionando-o com a satisfação no trabalho ${ }^{(3)} \mathrm{e}$ o desenvolvimento profissional ${ }^{(13)}$.

Entendendo-se que a tarefa do enfermeiro em liderar não é uma ação simples, pois envolve a complexa gestão da equipe de trabalho em prol de uma assistência mais eficaz ao paciente, conclui-se que o estudo sobre essa competência se faz cada vez mais necessário, para mapear como esta liderança tem ocorrido na prática clínica das instituições e seus reflexos na segurança do paciente e na satisfação profissional, pois autores afirmam que o exercício da liderança pode influenciar esses resultados ${ }^{(3)}$.

Levando em consideração a importância da liderança como competência essencial para o enfermeiro, surge a premência de compreender como os enfermeiros autoavaliam a liderança que exercem na prática e como os técnicos de enfermagem percebem essa liderança exercida pelo enfermeiro, além de compreender a influência dessa variável na satisfação no trabalho e na percepção da equipe quanto ao clima de segurança.

Frente a essas ponderações, surgiram as perguntas que guiaram a presente pesquisa: "Qual a autopercepção do enfermeiro quanto à sua prática de Liderança coaching?”, "Qual a percepção dos técnicos de enfermagem quanto à Liderança coaching exercida pelo enfermeiro?" " "A Liderança coaching influencia a satisfação no trabalho e o clima de segurança?".

As respostas a essas perguntas permitirão avançar no conhecimento sobre esse modelo de liderança, no que diz respeito à melhoria da qualidade da assistência e à satisfação profissional. Além disso, poderão subsidiar a gestão em enfermagem, customizando o desenvolvimento dessa competência a partir dos resultados encontrados. Logo, os objetivos desta pesquisa constituíram-se em comparar a percepção dos técnicos de enfermagem com a autopercepção do enfermeiro sobre o exercício da sua liderança coaching e verificar a influência desse modelo de liderança no clima de segurança e na satisfação da equipe.

\section{MÉTODO}

\section{Desenho do estudo}

Trata-se de um estudo transversal, quantitativo, descritivo e correlacional.

\section{CenÁrio}

A pesquisa foi realizada em um hospital escola, localizado em Campinas, SP, Brasil, que presta assistência em nível terciário, atende pacientes de alta complexidade e conta com 405 leitos. O hospital possui como missão a formação e a qualificação de recursos humanos e a produção do conhecimento científico. A equipe de enfermagem é constituída 
por 329 enfermeiros e 1.060 técnicos de enfermagem. No organograma da instituição, a diretora do departamento de enfermagem responde diretamente à superintendência do hospital. Abaixo dela, encontram-se os diretores de área, que recebem apoio dos supervisores, que são diretamente responsáveis por enfermeiros, e estes, pelos técnicos de enfermagem.

\section{Critérios de SEleÇÃO}

Como critérios de inclusão foram considerados os enfermeiros e os técnicos de enfermagem que atuavam diretamente na assistência e tinham, no mínimo, três meses de experiência. Esse tempo foi considerado pelo fato de ser o adotado como período mínimo necessário para efetivação de um profissional após sua contratação em grande parte das instituições hospitalares brasileiras. Por isso, acredita-se que, nesse período, o profissional já tenha se adaptado ao ambiente e conheça melhor os processos e a equipe com a qual trabalha. Foram excluídos os profissionais que estavam ausentes no período da coleta de dados, seja em decorrência de férias ou de licença médica, e aqueles que deixaram respostas em branco em qualquer um dos instrumentos.

\section{DEFINIÇÃO DA AMOSTRA}

O tamanho amostral foi calculado com base no poder do teste de $80 \%$, um nível de significância de 5\%, uma estimativa para o coeficiente de correlação igual a 0,30 e um coeficiente de correlação igual a 0,00 como hipótese nula. O cálculo resultou em uma amostra mínima de 168 profissionais (84 enfermeiros e 84 técnicos de enfermagem). Os enfermeiros foram aleatorizados a partir de planilhas do Microsoft Excel for Windows ${ }^{\circledR}$, nas quais constava o nome de todos os profissionais.

\section{Coleta de dados}

A coleta foi realizada no período entre outubro e dezembro de 2019, em uma única etapa e após a aleatorização. Os pesquisadores foram ao encontro dos enfermeiros em seus próprios locais e turnos de trabalho. Após a explicação dos objetivos do estudo, aqueles que aceitaram participar assinaram o Termo de Consentimento Livre e Esclarecido. Neste momento, também foi solicitada, ao enfermeiro, a escala dos técnicos de enfermagem de plantão naquele dia.

Dessa forma, os técnicos presentes também foram aleatorizados por meio de um site, acessado por smartphone. Aqueles que aceitaram participar receberam explicações sobre os objetivos do estudo e, dessa forma, foi constituída uma amostra pareada.

Para avaliar a Liderança coaching, foram utilizados o Questionário de Autopercepção do Enfermeiro no Exercício da Liderança (QUAPEEL), direcionado ao profissional enfermeiro, e o Questionário de Percepção do Técnico e Auxiliar de Enfermagem no Exercício da Liderança (QUEPTAEEL), direcionado aos técnicos de enfermagem. Ambos foram construídos e validados para uso no Brasil(16).

Os instrumentos QUAPEEL e QUEPTAELL são subdivididos em três partes. Para o presente estudo, foram utilizadas apenas duas partes: caracterização social e profissional da amostra, além de 20 itens, distribuídos em quatro dimensões: Comunicação, composta por cinco itens (1 a 5); Dar e receber feedback, composta por seis itens (6 a 11); Dar poder e exercer influência, composta por quatro itens (12 a 15), e Apoiar a equipe para o alcance dos resultados organizacionais, composta por cinco itens (16 a 20) ${ }^{(16)}$.

No que se refere à confiabilidade dos instrumentos, os coeficientes alfa de Cronbach obtidos para QUAPEEL e QUEPTAEEL foram, respectivamente 0,67 e 0,84, para a dimensão Comunicação; 0,71 e 0,81, para Dar e receber feedback; 0,74 e 0,89, para Dar poder e exercer influência; e 0,79 e 0,85, para Apoiar a equipe para o alcance dos resultados organizacionais.

Esses itens são avaliados por uma escala tipo Likert, com seis pontos, em que o valor um ponto representa "nunca não percebo a afirmação" e o valor cinco pontos representa "sempre - percebo todas as vezes a afirmação"; ou seja, quanto maior a pontuação obtida, maior é a percepção do exercício do comportamento descrito, na prática diária. $\mathrm{Na}$ escala de respostas, o participante também tem a opção da resposta "não se aplica". Os escores para as subescalas são obtidos pela média da soma das respostas dos profissionais ${ }^{(16)}$.

No que se refere à Satisfação no trabalho e ao Clima de segurança, os dados foram obtidos por meio das subescalas Clima de segurança e Satisfação no trabalho, do Questionário Atitudes de Segurança (SAQ), ambas validadas para uso no Brasil. A subescala Clima de Segurança $(\alpha=0,67)$ considera a percepção dos profissionais no que se refere ao comprometimento organizacional, para a segurança do paciente, sendo composta por sete itens. A subescala Satisfação no trabalho $(\alpha=0,77)$ reflete a visão positiva do profissional em relação ao seu ambiente de trabalho, sendo constituída por cinco itens, dos quais um item é reverso ${ }^{(17)}$. Estas subescalas foram aplicadas aos técnicos de enfermagem para checar se a percepção desses profissionais sobre a liderança exercida pelo enfermeiro (liderança real) tinha relação com o Clima de segurança e a Satisfação no trabalho.

A resposta para cada questão é avaliada por uma escala Likert com cinco opções, sendo A - discordo totalmente (0 pontos), $\mathrm{B}$ - discordo em parte ( 25 pontos), $\mathrm{C}-$ neutro (50 pontos), $\mathrm{D}$ - concordo em parte (75 pontos), $\mathrm{E}-$ concordo totalmente (100 pontos) e X - não se aplica (item deve ser excluído do cálculo da média). A pontuação final do instrumento varia de zero a 100 pontos, sendo 0 a pior avaliação e 100 a melhor. Pontuações acima de 75 indicam a percepção de um clima seguro para o paciente e de satisfação no trabalho. Para a realização do cálculo do escore, é necessário recodificar os itens reversos e, após, calcular a média da soma das respostas dos participantes em cada domínio ${ }^{(17)}$.

\section{AnÁlise e TRATAMENTO DOS DADOS}

Os dados obtidos foram tabulados em planilhas do Microsoft Excel for Windows ${ }^{\circledR}$, em que as frequências absolutas e relativas das variáveis categóricas e as medidas de posição das variáveis contínuas (média e desvio-padrão) foram calculadas. Profissionais que deixaram questões em branco (missing), em qualquer um dos instrumentos, foram excluídos da análise dos dados. 
Para as comparações entre os grupos, com relação aos escores dos instrumentos, foram aplicados os testes MannWhitney. As correlações foram avaliadas por meio do coeficiente de correlação de Spearman, cujos valores entre 0,1 e 0,29 são considerados de fraca magnitude, de 0,30 a 0,49 , de moderada, e aqueles maiores ou iguais a 0,50 , de forte magnitude $^{(18)}$. O nível de significância adotado foi de $5 \%$. Para todas as análises, foi utilizado o software estatístico Statistical Analysis Software ${ }^{\circledR}$ (SAS) versão 9.4.

\section{AsPeCTOS ÉTICOS}

O estudo foi submetido à apreciação e à aprovação do Comitê de Ética em Pesquisa da Universidade, sob o protocolo de número 2.202.970. Cabe informar que, no desenvolvimento da pesquisa, foram respeitados os princípios éticos estabelecidos pela Resolução no 466/2012, do Conselho Nacional de Saúde, que trata de pesquisas envolvendo seres humanos. Foi assegurado o anonimato dos participantes.

\section{RESULTADOS}

Foram convidados para participar da pesquisa 170 profissionais de enfermagem, sendo 85 enfermeiros e 85 técnicos. Dentre os participantes, alguns não preencheram os campos relacionados à idade e ao tempo na função e na unidade. $\mathrm{Na}$ Tabela 1, encontram-se os dados que possibilitam o conhecimento de um perfil mais detalhado dos participantes.

Tabela 1 - Variáveis de caracterização da amostra - Campinas, SP, Brasil, 2019.

\begin{tabular}{|c|c|c|c|c|c|c|c|c|}
\hline \multirow{2}{*}{ Variáveis } & \multicolumn{4}{|c|}{ Enfermeiros } & \multicolumn{4}{|c|}{ Técnicos } \\
\hline & $\mathbf{n}$ & $\%$ & Média & DP* & $\mathbf{n}$ & $\%$ & Média & DP* \\
\hline Idade & 79 & & 41,0 & 10,6 & 76 & & 38,1 & 10,2 \\
\hline Tempo na função & 80 & & 12,9 & 10,1 & 74 & & 14,2 & 9,2 \\
\hline Tempo na unidade & 78 & & 8,0 & 8,2 & 71 & & 8,0 & 6,8 \\
\hline \multicolumn{9}{|l|}{ Sexo } \\
\hline Feminino & 73 & 85,9 & & & 59 & 69,4 & & \\
\hline Masculino & 12 & 14,1 & & & 25 & 29,4 & & \\
\hline Outros & - & - & & & 1 & 1,1 & & \\
\hline \multicolumn{9}{|l|}{ Turno } \\
\hline Manhã & 20 & 23,5 & & & 20 & 23,5 & & \\
\hline Tarde & 26 & 30,6 & & & 26 & 30,6 & & \\
\hline Noite & 39 & 45,9 & & & 39 & 45,9 & & \\
\hline \multicolumn{9}{|l|}{ Setor de Trabalho } \\
\hline Unidades de Terapia Intensiva & 31 & 36,5 & & & 31 & 46,7 & & \\
\hline Unidades de Internação & 30 & 35,2 & & & 30 & 35,2 & & \\
\hline Unidades de Emergência & 20 & 23,5 & & & 12 & 23,5 & & \\
\hline Centro Cirúrgico & 4 & 4,7 & & & 4 & 4,7 & & \\
\hline \multicolumn{9}{|l|}{ Formação profissional } \\
\hline Técnico & - & - & & & 67 & 78,8 & & \\
\hline Graduação & 36 & 42,3 & & & 11 & 12,9 & & \\
\hline Pós Lato Sensu & 43 & 50,6 & & & 7 & 8,2 & & \\
\hline Pós Stricto Sensu & 6 & 7,1 & & & - & - & & \\
\hline
\end{tabular}

*DP: Desvio Padrão

$\mathrm{Na}$ Tabela 2, são apresentadas a avaliação da autopercepção do enfermeiro, quanto ao exercício da sua liderança, a avaliação da percepção dos técnicos, quanto à liderança exercida pelo enfermeiro, bem como a comparação entre essas avaliações considerando o pareamento entre enfermeiros e técnicos. É possível notar, nessa tabela, que, nas dimensões Comunicação, Dar e receber feedback, Dar poder e exercer influência oito participantes foram excluídos da análise. Já na dimensão Apoiar a equipe para $o$ alcance dos resultados organizacionais, 11 participantes foram excluídos, em decorrência de missings.

Tabela 2 - Comparação entre a auto percepção do enfermeiro quanto ao exercício da sua liderança e a percepção dos técnicos quanto à liderança exercida pelo enfermeiro - Campinas, SP, Brasil, 2019.

\begin{tabular}{|c|c|c|c|c|c|}
\hline Dimensões & Categoria & $\mathbf{n}$ & Média & DP* & p valor $t$ \\
\hline \multirow{2}{*}{ Comunicação } & Enfermeiro & 77 & 4,30 & 0,42 & \multirow{2}{*}{0,1504} \\
\hline & Técnico & 77 & 4,20 & 0,70 & \\
\hline \multirow{2}{*}{ Dar e receber feedback } & Enfermeiro & 77 & 4,30 & 0,50 & \multirow{2}{*}{0,0036} \\
\hline & Técnico & 77 & 3,90 & 0,90 & \\
\hline \multirow{2}{*}{ Dar poder e exercer influência } & Enfermeiro & 77 & 4,23 & 0,55 & \multirow{2}{*}{$<0,0001$} \\
\hline & Técnico & 77 & 3,68 & 1,02 & \\
\hline \multirow{2}{*}{ Apoiar a equipe para o alcance dos resultados organizacionais } & Enfermeiro & 74 & 4,15 & 0,68 & \multirow{2}{*}{$<0,0001$} \\
\hline & Técnico & 74 & 3,54 & 1,00 & \\
\hline
\end{tabular}

Desvio padrão; + Valor de p obtido por meio do teste de Mann-Whitney. 
A correlação entre as dimensões Clima de segurança e Satisfação no trabalho, do $\mathrm{SAQ}_{2}$ e a percepção dos técnicos sobre o exercício da liderança exercida pelos enfermeiros é demonstrada na Tabela 3.

Tabela 3 - Correlação entre a percepção dos técnicos de enfermagem sobre a liderança exercida pelo enfermeiro e as subescalas Clima de segurança e Satisfação no trabalho, Campinas, SP, Brasil, 2019.

\begin{tabular}{|c|c|c|}
\hline QUEPTAEEL & Clima de segurança & Satisfação no Trabalho \\
\hline Comunicação & $\begin{array}{c}0,2984 \\
\mathbf{0 , 0 0 6 8} *\end{array}$ & $\begin{array}{c}0,1831 \\
0,1017^{*}\end{array}$ \\
\hline Dar e receber feedback & $\begin{array}{c}0,3965 \\
\mathbf{0 , 0 0 0 2}\end{array}$ & $\begin{array}{c}0,2596 \\
\mathbf{0 , 0 1 9 3}\end{array}$ \\
\hline Dar poder e exercer influência & $\begin{array}{c}0,4776 \\
<\mathbf{0 , 0 0 0 1} \text { * }\end{array}$ & $\begin{array}{c}0,3979 \\
\mathbf{0 , 0 0 0 3 *}\end{array}$ \\
\hline Apoiar a equipe para o alcance dos resultados organizacionais & $\begin{array}{c}0,3744 \\
\mathbf{0 , 0 0 0 9} *\end{array}$ & $\begin{array}{c}0,3173 \\
\mathbf{0 , 0 0 5 2}\end{array}$ \\
\hline
\end{tabular}

*Valores de p obtidos por meio do coeficiente de correlação de Spearman.

Na Tabela 3, é possível observar correlações significantes, positivas e de moderada magnitude entre Clima de segurança e Dar e receber feedback $(\mathrm{r}=0,39 ; \mathrm{p}=0,0002)$; Dar poder e exercer influência $(r=0,47 ; p<0,0001)$, e Apoiar a equipe para o alcance dos resultados organizacionais $(\mathrm{r}=0,37 ; \mathrm{p}=0,0009)$. Também foram apresentadas correlações positivas e de moderada magnitude entre Satisfação no trabalho e Dar poder e exercer influência $(r=0,39$; $p=0,0003$ ), Apoiar a equipe para o alcance dos resultados organizacionais $(r=0,31 ; p=0,0052)$.

\section{DISCUSSÃO}

O último relatório do grupo parlamentar de saúde global, Triple Impact of Nursing, demonstrou que a enfermagem necessita de líderes qualificados que estimulem suas equipes a atingir o máximo do seu potencial ${ }^{(19)}$.

Frente a esse cenário, a ferramenta coaching destaca-se como um importante modelo de liderança qualificada, que contribui para o desenvolvimento tanto da prática clínica quanto da gestão de pessoas. Por isso, a avaliação das dimensões do processo coaching se faz cada vez mais necessária para o direcionamento inovador das organizações de saúde $\mathrm{d}^{(3,12-13)}$.

Neste estudo, ao se comparar a percepção dos enfermeiros e dos técnicos de enfermagem quanto ao exercício da Liderança coaching, foi possível notar que o enfermeiro teve melhor percepção do desempenho da sua liderança do que o técnico sobre o exercício de liderança do enfermeiro, em todas as dimensões, sendo que, em três delas (Dar e receber feedback, Dar poder e exercer influência e Apoiar a equipe para o alcance dos resultados organizacionais), essa diferença foi significante.

Pesquisadores encontraram resultados semelhantes aos da presente pesquisa, em que a autoavaliação dos enfermeiros alcançou maiores médias nas dimensões Dar e receber feedback, Dar poder e exercer influência e Apoiar a equipe para o alcance dos resultados organizacionais, quando comparados aos da percepção dos técnicos e dos auxiliares de enfermagem ${ }^{(13)}$. Tal fato reforça que os técnicos avaliaram que não estão recebendo o acompanhamento periódico do seu desempenho, ou notando influência no desenvolvimento de suas competências e o apoio na resolução de dificuldades da mesma forma que os enfermeiros avaliaram o desempenho da sua liderança ${ }^{(16,20)}$.

Estudos vêm demonstrando uma relação positiva entre feedback e aumento da cultura de segurança no ambiente de saúde, melhoria da qualidade do cuidado, desenvolvimento de habilidades técnicas dos profissionais, diminuição da exaustão emocional dos liderados e menor intenção dos profissionais deixarem seus empregos. Portanto, o feedback constitui uma importante dimensão que pode contribuir para resultados positivos para pacientes, profissionais e instituições; por isso, precisa ser valorizado e, efetivamente, implementado ${ }^{(21-22)}$.

Com relação à dimensão Dar poder e exercer influência, os resultados deste estudo mostraram que o enfermeiro precisa rever sua percepção quanto ao compartilhamento do poder. Dar poder e receber influência tem relação com a participação dos liderados junto à gestão e com o compartilhamento de responsabilidades com o intuito de alcançar resultados mais favoráveis ${ }^{(16)}$. A atuação do enfermeiro torna-se mais significativa para a equipe quando as decisões são tomadas em conjunto ${ }^{(13)}$, pois, dessa forma, os liderados sentem-se mais valorizados e, consequentemente, mais satisfeitos e motivados para o desenvolvimento do trabalho ${ }^{(23)}$.

Sobre a dimensão Apoiar a equipe para o alcance dos resultados organizacionais, assim como relatado por outros autores $^{(13)}$, foi possível perceber que os enfermeiros ainda precisam aprimorar sua capacidade de incentivo e apoio às equipes, visando ao alcance de metas para que uma relação de abertura e confiança possa ser estabelecida no ambiente de trabalho.

A respeito da Comunicação, destaca-se que essa dimensão foi a que obteve maiores médias entre os profissionais, não tendo sido evidenciadas diferenças significantes entre os grupos. Esse resultado é positivo, pois uma comunicação eficaz colabora para a redução do estresse, a promoção do bem-estar da equipe e a melhora da qualidade de vida dos trabalhadores ${ }^{(3)}$, além de melhorar a segurança do paciente ${ }^{(24)}$.

Outro aspecto importante desta pesquisa foi a avaliação da relação entre a percepção dos técnicos de enfermagem sobre a liderança exercida pelos enfermeiros e a percepção do Clima de segurança. Foi possível perceber que, quanto mais os 
aspectos das dimensões coaching são exercidos e, dessa forma, percebidos pelos técnicos de enfermagem, melhor também é o clima de segurança que permeia a assistência ao paciente.

Outros estudos avaliaram a influência de diferentes modelos de liderança sobre o clima de segurança ${ }^{(25-26)}$ e revelaram que a liderança exercida pelo enfermeiro é essencial para fornecer uma assistência de alta qualidade ao paciente ${ }^{(7,23,27)}$ e garantir resultados organizacionais favoráveis ${ }^{(7)}$.

Ao relacionar a percepção dos técnicos quanto à liderança exercida pelo enfermeiro e a variável Satisfação no trabalho, também se observou que quanto mais o enfermeiro implementar as dimensões da liderança coaching, mais os profissionais se sentirão satisfeitos com os seus empregos.

Corroborando esses achados, verificou-se que outros pesquisadores também encontraram que a inspiração, a motivação ${ }^{(3,13)}$, a confiança mútua, a interação contínua entre líder e liderados e a busca pelo desenvolvimento profissional e pessoal são essenciais para a satisfação dos profissionais ${ }^{(3)}$.

Outro estudo revelou que o modelo de liderança pode influenciar no fortalecimento do local de trabalho, o que, por sua vez, aumenta a satisfação dos enfermeiros no trabalho e diminui a frequência de resultados adversos para os pacientes ${ }^{(28)}$.

Estudiosos apontam que, quando o enfermeiro mantém uma relação mais próxima com a equipe, apoiando-a para o alcance dos resultados organizacionais, valorizando a comunicação efetiva, realizando o feedback e exercendo uma influência positiva, melhores resultados são alcançados ${ }^{(29-30)}$.

Os enfermeiros do presente estudo não foram capacitados para a liderança coaching. Considerando-se que esse modelo possibilita o desenvolvimento profissional, recomenda-se que a abordagem do tema seja feita desde o curso de graduação, pois a enfermagem, profissão dinâmica e desafiadora, exige líderes que envolvam e inspirem seus liderados.
No atual ambiente de saúde, identificar e desenvolver líderes é um dos maiores desafios enfrentados pelos gestores, pois, para o alcance de melhores resultados, a enfermagem necessita de profissionais cada vez mais qualificados.

Em vista disso, este estudo pode ser considerado um importante meio para o estímulo à prática da Liderança coaching. Além do mais, o estudo disponibiliza informações que podem auxiliar as instituições a perceberem que a liderança qualificada baseada em comunicação efetiva, feedback, influência e apoio é uma ferramenta de fundamental importância na busca da satisfação no trabalho e da qualidade da assistência oferecida ao paciente.

Como limitação do estudo, destaca-se que a análise da comparação das percepções da equipe de enfermagem ocorreu em um contexto, portanto, caracterizando percepções de uma população específica. Além disso, ao considerar os critérios de exclusão, o número total de participantes acabou não atingindo o tamanho amostral calculado inicialmente. Sendo assim, novos estudos sobre a temática são necessários, para ampliar o conhecimento, bem como a consciência do enfermeiro em relação à sua prática de liderança.

\section{CONCLUSÃO}

A autopercepção dos enfermeiros com relação ao exercício da sua liderança foi mais positiva do que a avaliação dos técnicos sobre o exercício da liderança pelo enfermeiro, especialmente nas dimensões Dar e receber feedback, Dar poder e exercer influência e Apoiar a equipe para o alcance dos resultados organizacionais. Quanto mais o enfermeiro exercer as dimensões da Liderança coaching e, consequentemente, isso for percebido pela equipe, melhor serão o clima de segurança e a satisfação da equipe com relação ao seu trabalho.

\section{RESUMO}

Objetivo: Comparar a percepção dos técnicos de enfermagem com a autopercepção do enfermeiro sobre o exercício da sua Liderança coaching e verificar a influência desse modelo de liderança no clima de segurança e na satisfação da equipe. Método: Estudo correlacional, realizado com 85 enfermeiros e 85 técnicos de enfermagem, utilizando o Questionário de Autopercepção do Enfermeiro no Exercício da Liderança, o Questionário de Percepção do Técnico e Auxiliar de Enfermagem no Exercício da Liderança e as subescalas Clima de segurança e Satisfação no trabalho. As relações entre as respostas dos profissionais foram avaliadas pelo teste de Mann Whitney e coeficiente de Spearman. Resultados: Os enfermeiros alcançaram maiores médias nas quatro dimensões da Liderança coaching, sendo que, em três, as diferenças foram significantes $(\mathrm{p}<0,05)$. As dimensões da Liderança coaching obtiveram correlações positivas e significantes com as subescalas Clima de segurança e Satisfação, sendo a maioria de moderada magnitude. Conclusão: A autopercepção dos enfermeiros, com relação ao exercício da liderança, foi mais positiva do que a avaliação dos técnicos. Quanto mais o enfermeiro exercer as dimensões da Liderança coaching, melhores serão o clima de segurança e a satisfação da equipe.

\section{DESCRITORES}

Liderança; Supervisão de Enfermagem; Satisfação no Emprego; Segurança do Paciente; Papel do Profissional de Enfermagem; Administração de Serviços de Saúde.

\section{RESUMEN}

Objetivo: Comparar la percepción de los técnicos de enfermería con la autopercepción del enfermero sobre la práctica de su Liderazgo coaching y verificar la influencia de ese modelo de liderazgo en el clima de seguridad y en la satisfacción del equipo. Método: Estudio correlacional, realizado con 85 enfermeros y 85 técnicos de enfermería que utilizaron el Cuestionario de Autopercepción del Enfermero en la Práctica del Liderazgo, el Cuestionario de Percepción del Técnico y Auxiliar de Enfermería en la Práctica del Liderazgo y las subescalas clima de seguridad y Satisfacción en el trabajo. Las relaciones entre las respuestas de los profesionales fueron evaluadas por el test de Mann Whitney y coeficiente de Spearman. Resultados: Los enfermeros alcanzaron mejores medias en las cuatro dimensiones del Liderazgo coaching, siendo que, en tres, las diferencias fueron significativas $(p<0,05)$. Las dimensiones del Liderazgo coaching obtuvieron correlaciones positivas y significativas con las subescalas Clima de seguridad y satisfacción, siendo la mayoría de moderada magnitud. Conclusión: La autopercepción de los enfermeros, con relación a la práctica del liderazgo, fue más positiva que la evaluación de los técnicos. A la medida que el enfermero ejerce más las dimensiones de Liderazgo coaching, mejores serán el clima de seguridad y la satisfacción del equipo. 


\section{DESCRIPTORES}

Liderazgo; Supervisión de Enfermería; Satisfacción en el Trabajo; Seguridad del Paciente; Rol de la Enfermera; Health Services Administration.

\section{REFERÊNCIAS}

1. Roobbins SP, Judge TA, Sobral F. Comportamento organizacional: teoria e prática no contexto brasileiro. São Paulo: Pearson, 2010.

2. Brasil Ministério da Saúde. Conselho Nacional de Saúde. Resolução n 573, de 31 de janeiro de 2018. Dispõe sobre homologação nos termos do Decreto de Delegação de Competência de 12 de novembro de 1991. Diário Oficial União, Brasília, DF. 2018 Nov 11;Section 1:38.

3. Moura AA, Bernardes A, Balsanelli AP, Dessotte CAM, Gabriel CS, Zanetti ACB. Leadership and job satisfaction in the mobile emergency care service context. Rev Lat Am Enfermagem. 2020[cited 2020 Jun 24]; 28:e3260. https://doi.org/10.1590/1518-8345.3455.3260

4. Montezeli JH, Almeida KP, Haddad MCFL. Nurses' perceptions about social skills in care management from the perspective of complexity. Rev Esc Enferm USP. 2018;52:e03391.

5. Lee E, Daugherty J, Hamelin T. Reimagine health care leadership, challenges and opportunities in the 21st century. J Perianesth Nurs. 2019;34(1):27-38. https://doi.org/10.1016/j.jopan.2017.11.007

6. Boamah AS, Lashinger HKS, Wong C, Clark S. Effect of transformational leadership on job satisfaction and patient safety outcomes. Nurs Outlook. 2018;66(2):180-9. https://doi.org/10.1016/j.outlook.2017.10.004

7. Inoue T, Karima R, Harada K. Bilateral effects of hospital patient-safety procedures on nurses' job satisfaction. Int Nurs Rev. 2017;64(3):43745. https://doi.org/10.1111/inr.12336

8. Sola GD, Badia JG, Hito D, Obasa AC, Garcia JLDV. Self-perception of leadership styles and behaviour in primary health care. BMC Health Serv Res 2016;16:572. https://doi.org/10.1186/s12913-016-1819-2

9. Oliveira C, Santos LC, Andrade J, Domingos TS, Spiri WC. Leadership in the perspective of Family Health Strategy nurses. Rev Gaúcha Enferm. 2020;41:e20190106. https://doi. org/10.1590/1983-1447.2020.20190106

10. Mondini CCSD, Cunha ICKO, Trettene ADS, Fontes CMB, Bachega MI, Cintra FMR. Authentic leadership among nursing professionals: knowledge and profile. Rev Bras Enferm. 2020;73(4):1-7. https://doi.org/10.1590/0034-7167-2018-0888

11. Ortiz RD, Neto ATS. Coaching leadership: current and future perspectives in nursing. Braz J Health Rev. 2019 [cited 2020 Jun 23];2(1):262-76. Available from: https://www.brazilianjournals.com/index.php/BJHR/article/view/925

12. Jennifer $M B$, Weaver. The perceptions of professional leadership coaches regarding the roles and challenges of nurse managers. J Nurs Adm. 2019;49(2):105-9. https://doi.org/10.1097/NNA.0000000000000718

13. Hayashida KY, Bernardes A, Moura AA, Gabriel CS, Balsanelli AP. Coaching leadership exercised by nurses in the hospital setting. Cogitare Enferm. 2019;24:e59789. http://dx.doi.org/10.5380/ce.v24i0.59789

14. YuKyung K, Soyoung Y. The Relationships among perceived patients' safety culture, intention to report errors, and leader coaching behavior of nurses in Korea: a pilot study. J Patient Saf. 2017;13(3):175-83. DOI: https://doi.org/10.1097/PTS.0000000000000224

15. Cardoso MLAP, Ramos LH, D’Innocenzo M. Liderança coaching: um modelo de referência para o exercício do enfermeiro-líder no contexto hospitalar. Rev Esc Enferm USP. 2011;45(3):730-37. DOI: https://doi.org/10.1590/S0080-62342011000300026

16. Cardoso MLPA, Ramos LH, D 'Innocenzo M. Coaching leadership: leaders' and followers' perception assessment questionnaires in nursing Einstein. 2014;12(1):66-74. https://doi.org/10.1590/S1679-45082014AO2888

17. Carvalho REFL, Cassiani SHB. Cross-cultural adaptation of the Safety Attitudes Questionnaire - Short Form 2006 for Brazil. Rev Lat Am Enfermagem. 2012;20(3):575-82. https://doi.org/10.1590/S0104-11692012000300020

18. Cohen J. Statistical power analysis for the behavioral sciences. 2nd ed. New Jersey: Lawrence Erlbaum; 1988. The significance of a product moment; p. 75-108.

19. All-Party Parliamentary Group on Global Health. Triple impact: how developing nursing will improve health, promote gender equality and support economic growth. Geneve: All-Party Parliamentary Group on Global Health 2016 [cited 2020 Jun 22]. Available from: https://www.who.int/hrh/com-heeg/digital-APPG_triple-impact.pdf?ua=1

20. Akeriordet K, Furunes T, Haver A. Health-promoting leadership: an integrative review and future research agenda. J Adv Nurs. 2018;74(7):1505-16. https://doi.org/10.1111/jan.13567

21. Silva VLS, Camelo SHH, Soares MI, Resck ZMR, Chaves LDP, Santos FC, et al. Leadership practices in hospital nursing: a self of manager nurses. Rev Esc Enferm USP. 2017;51:e03206. https://doi.org/10.1590/S1980-220X2016024403206

22. Sexton IJ, Adair KC, Leonard MW, Frankel TC, Proulx J, Watson SR, et al. Providing feedback following leadership walkrounds is associated with better patient safety culture, higher employee engagement and lower burnout. BMJ Qual Saf. 2018;27(4):261-70. https://doi.org/10.1136/bmjqs-2016-006399

23. Sfantou DF, Laliotis A, Patelarou AE, Pistolla DS, Matalliotakis M, Patelarou E. Importance of leadership style towards quality of care measures in healthcare settings: a systematic review. Healthcare. 2017;5(4):1-17. https://doi.org/10.3390/healthcare5040073

24. Kim KJ, Yoo MS, Seo EJ. Exploring the influence of nursing work environment and patient safety culture on missed nursing care in Korea. Asian Nurs Res. 2018;12(2):121-26. https://doi.org/10.1016/j.anr.2018.04.003

25. Dirik HF, Intepeler SS. The influence of authentic leadership on safety climate in nursing. J Nurs Manag. 2017;25(5):392-401. https://doi.org/10.1111/jonm.12480

26. Amany F, McGuinness TS, Mary AK, Burant C. Do leadership style, unit climate, and safety climate contribute to safe medication practices? J Nurs Adm. 2017;47(1):8-15. https://doi.org/10.1097/NNA.0000000000000430 
27. Kristensen S, Cristensen KB, Jaquet A, Beck CM, Sabroe S, Bartels P, Mainz J. Strengthening leadership as a catalyst for enhanced patient safety culture: a repeated cross-sectional experimental study. BMJ Open 2016;6:e010180. https://doi.org10.1136/bmjopen-2015- 010180

28. Heather K. Laschinger HKS, Wong C, Clarke S. Effect of transformational leadership on job satisfaction and patient safety outcomes. Nurs Outlook. 2018;66(2):180-9. https://doi.org/10.1016/j.outlook.2017.10.004

29. Mello JF, Barbosa SFF. Patient safety culture in an intensive care unit: the perspective of the nursing team. Rev Eletrônica Enferm. 2017;19:a07. https://doi.org/10.5216/ree.v19.38760

30. Broca PV, Ferreira MA. Communication process in the nursing team based on the dialogue between Berlo and King. Esc Anna Nery. 2015;19(3):467-74. https://doi.org/10.5935/1414-8145.20150062 